\title{
Novel Base Station MIMO Antennas with Enhanced Spectral Efficiencies Using Angular Reuse
}

\author{
Miguel Mora-Andreu ${ }^{1}$ and David A. Sánchez-Hernández ${ }^{2}$ \\ ${ }^{1}$ EMITE Ingeniería S.L., 30320 Fuente Álamo de Murcia, Spain \\ ${ }^{2}$ Universidad Politécnica de Cartagena, 30202 Cartagena, Spain \\ Correspondence should be addressed to Miguel Mora-Andreu; miguel.mora@emite-ingenieria.es
}

Received 4 December 2014; Revised 12 May 2015; Accepted 12 May 2015

Academic Editor: Heng-Tung Hsu

Copyright ( 2015 M. Mora-Andreu and D. A. Sánchez-Hernández. This is an open access article distributed under the Creative Commons Attribution License, which permits unrestricted use, distribution, and reproduction in any medium, provided the original work is properly cited.

\begin{abstract}
The true polarization diversity (TPD) technique is combined with the spatial diversity technique in novel MIMO antenna array geometries with a large number of elements. The use of a large number of elements requires some angular reuse within the array for polarization diversity. With designs compatible with existing base station antenna array configurations, the novel geometries with combining diversity schemes are shown to be able to achieve near the maximum spectral efficiencies. True polarization diversity (TPD) schemes are found to be an excellent complement to more conventional spatial diversity schemes for obtaining optimum MIMO array performance in base station antennas.
\end{abstract}

\section{Introduction}

The application of MIMO techniques $[1,2]$ in user wireless terminals is now a reality. An element having a strong effect on final measured spectral efficiencies in practice and that has received strong attention in the literature is the antenna efficiency [3-5]. The use of MIMO techniques in the base station antenna array, however, is still very limited considering that the base station side does not have the strict volume limitations that exist on the UE size. Enhancements are being developed on the use of multiple carriers (carrier aggregation) with the same $2 \times 2$ MIMO scheme, therefore exploiting the spectrum domain, rather than further exploiting the spatial-angular domain. The basic principle of diversity in wireless communication systems is that MIMO transmitted signals experience different signal fading due to different channel conditions, and these different signals are only partially correlated, something which therefore can be exploited upon reception by diverse techniques (selection combining, maximum ratio combining, etc.). The aim is to get some gain over the situation in which no diversity is employed, that is, similar throughput values for lower signal to noise ratios. For MIMO modes, the idea is to exploit the different eigenvalues existing on the channel to obtain enhanced throughput values for the same signal to noise ratios. In multipath propagation conditions each received antenna experiences a different fading environment. In consequence, multiple diversity dimensions can be exploited in receivers, and this is in fact a commonly used technique in current commercial user equipment- (UE-) integrated antenna MIMO arrays.

Among the possible diversity variations, polarization diversity $[5,6]$ was recently extended beyond the concept of orthogonal polarization diversity (OPD) in which only two orthogonal polarization states were used to a much more general true polarization diversity (TPD) [5] in which, in MIMO antenna array, several polarization states can be effectively reused for obtaining larger capacities. The idea is that the correlation between consecutive elements can be minimized beyond the use of orthogonal states, resulting in MIMO combinations which were found to be as effective as spatial diversity in [5] and a strong potential candidate for future base station antenna designs when combined with spatial diversity. TPD designs were found to start outperforming conventional OPD designs with as low as three antenna elements in the array. 
In this contribution, the potential of the combined TPD and the spatial diversity techniques is further demonstrated for obtaining large capacities with reduced array dimensions and a large number of antennas in novel geometries suitable for integration in existing base station antenna arrays. Results will show that the combined spatial-TPD technique employing efficient angular reuse provides for enhanced capacities, only limited by the limited number of antennas. Moreover, it will be concluded that, for specific scenarios, polarization diversity or spatial diversity only schemed may be more efficient for final enhanced base station MIMO antenna performance.

The paper is organized as follows. Section 2 describes and validates the developed model for obtaining the spatialangular combined correlation function. Section 3 provides combined results for both spatial and angular diversity combinations with reuse of polarization states. Section 4 analyzes the obtained results. Finally, conclusions are outlined in Section 5 .

\section{Correlation Model}

To fully characterize the potential diversity between two antennas, the correlation function between them has to be studied. A method which is extensively used for measuring the correlation coefficient between two antennas is the reverberation chamber (RC) [7-9], which has already shown that a correlation coefficient below 0.5 can hardly benefit the final measured capacity beyond what is obtained at 0.5 [10]. In this contribution, the correlation between two dipoles separated an arbitrary spatial and angular distance will be studied. The spatial correlation function for two parallel dipoles is well known in the literature, and the spatial correlation function of two dipoles with both a spatial separation of $d$ wavelengths and an angular separation of $\theta$ degrees was presented in [11].

In this section the spatial, angular, and spatial-angularcombined correlation functions are estimated and validated using measurements in a commercial reverberation chamber.

2.1. Spatial Correlation Model. The model typically used to calculate the spatial correlation among antennas is well known and it is described in [1] and has been previously validated for its use in a reverberation chamber in [2-4]. The spatial correlation $\zeta s$ between two antennas is defined as

$$
\zeta_{\mathrm{spa}}=J_{0}\left[z_{\mathrm{spa}}^{i-k}\right]
$$

where $J_{0}$ is the zeroth-order Bessel functions of the first kind, $z_{\text {spa }}^{i-k}=2 * \pi * d_{\text {spa }} / \lambda, d_{\text {spa }}$ is the interelement spatial distance, and $\lambda$ is the wavelength.

2.2. Angular Correlation Model. An empirical model is proposed for the estimation of the angular correlation between two antennas. In this model, the angular separation is considered to be equivalent to the spatial separation between antennas, and therefore an equivalent angular distance $d_{\text {ang }}$ can be defined as

$$
d_{\mathrm{ang}}=\left[\left(\varphi^{\prime}{ }_{i-k}\right)\right]
$$

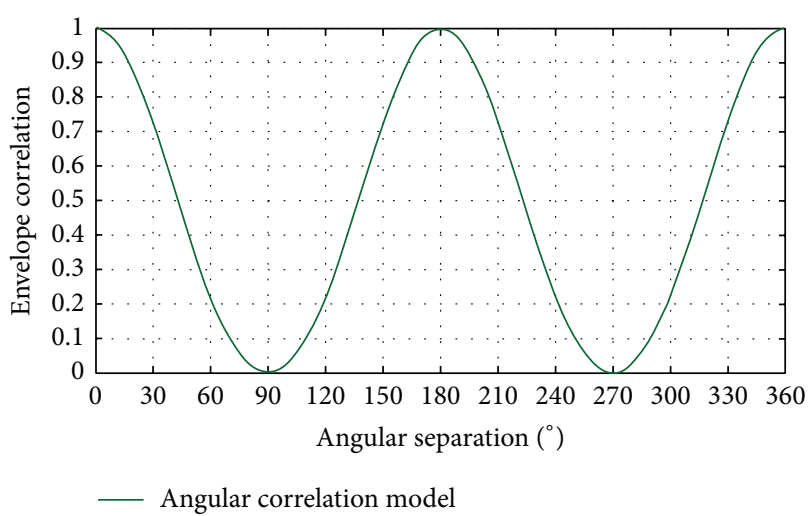

Figure 1: Angular correlation for two dipole antennas using the empirical model.

where $\varphi^{\prime}{ }_{i-k}$ is a function defined by

$$
\begin{aligned}
& \varphi_{i-k}^{\prime} \\
& =\left\{\begin{array}{c}
\varphi_{i-k}=\operatorname{abs}\left(\varphi_{i-k}\right) \longrightarrow \operatorname{abs}\left(\varphi_{i-k}\right) \leq 90 \\
\varphi_{i-k}=180-\operatorname{abs}\left(\varphi_{i-k}\right) \longrightarrow 90<\operatorname{abs}\left(\varphi_{i-k}\right) \leq 270 \\
\varphi_{i-k}=360-\operatorname{abs}\left(\varphi_{i-k}\right) \longrightarrow 270<\operatorname{abs}\left(\varphi_{i-k}\right) \leq 360
\end{array}\right\},
\end{aligned}
$$

where $\varphi_{i-k}$ is the interelement angular distance. Thus, the angular correlation would be similar to

$$
\zeta_{a}=J_{0}\left\lfloor z_{\mathrm{eq}}\right\rfloor
$$

where

$$
z_{\mathrm{eq}}=\frac{2 * \pi * d_{\mathrm{eq}}}{\lambda}
$$

The envelope correlation coefficient versus angular separation estimated using this empirical model for a pair of halfwave dipole antennas is illustrated in Figure 1.

In order to validate the angular correlation model, the correlation matrix of a pair of antennas with angular separations was measured on an EMITE E400 mode-stirred source-stirred MIMO analyzer reverberation chamber. This commercial system has been validated by 3GPP and CTIA (see Appendix A in [12]). The EMITE E400 MIMO analyzer reverberation chamber consists of a compact reverberation chamber $(2.70 \times 2.00 \times 1.55 \mathrm{~m})$ with eight source antennas (source-stirring), three metallic mode-stirrers (modestirring), and a turntable (platform-stirring), with $\sim 690 \mathrm{MHz}$ lowest frequency of operation. The test setup is depicted in Figure 2.

Correlation measurements were performed while a dipole was rotated with respect to the other from 0 to $360^{\circ}$ in $10^{\circ}$ steps. Measurements were performed at $900 \mathrm{MHz}$ and half-wave dipoles were used as MIMO array antennas. Results, depicted in Figure 3, show a good matching between the angular correlation model and measured results. 


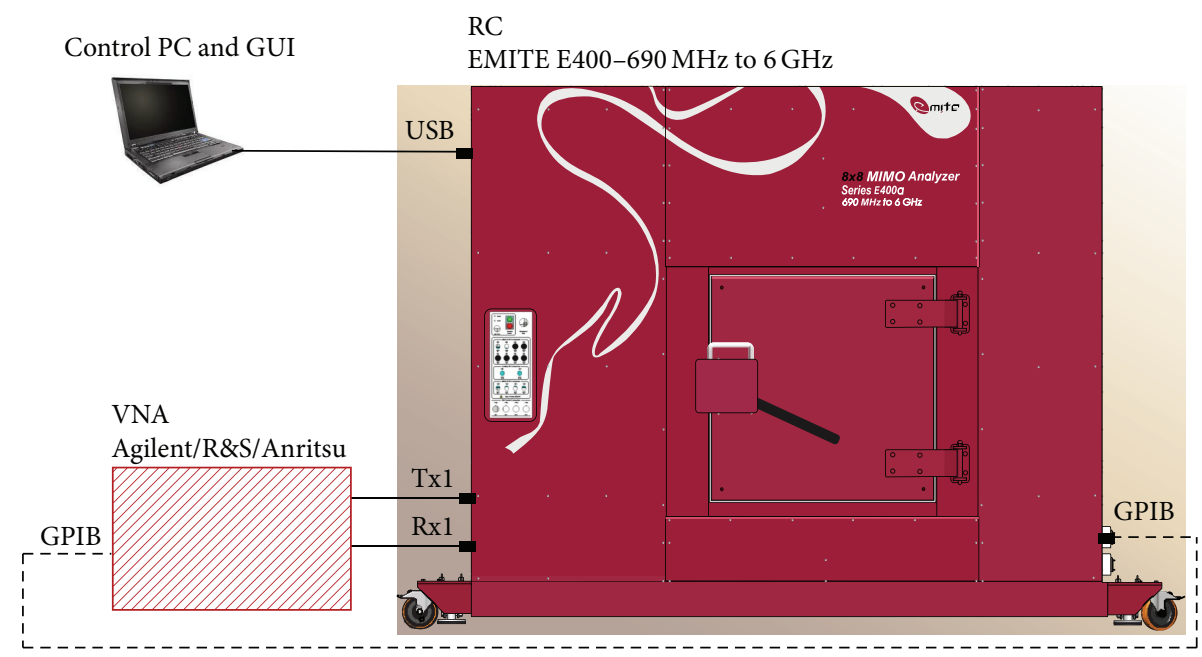

FIGURE 2: E400 MIMO analyzer reverberation chamber test setup.

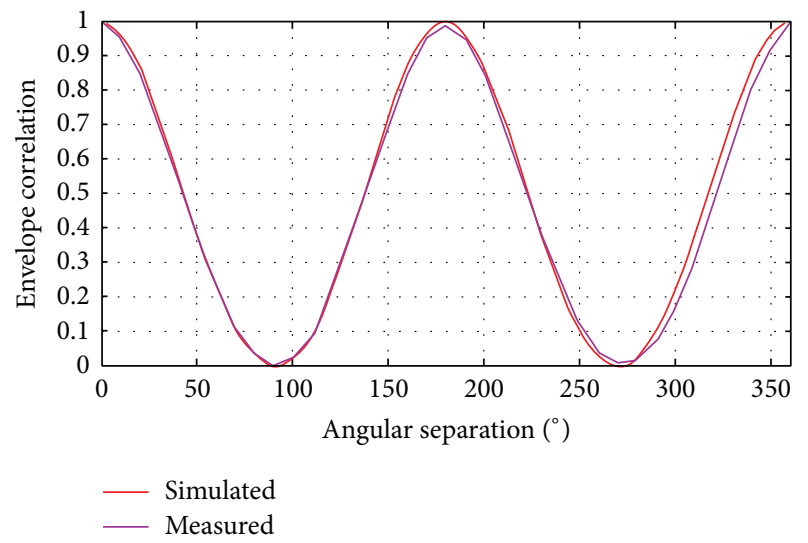

FIGURE 3: Envelope correlation of a pair of $\lambda / 2$ dipole antennas as a function of their angular separation. Simulations and measurements.

2.3. Combined Correlation Model. The spatial correlation model and the angular correlation model validated above can be combined by adding the spatial and angular equivalent distances, so that

$$
\zeta=J_{0}\left[z_{\text {total }}\right]
$$

where

$$
z_{\text {total }}=\frac{2 * \pi *\left(d_{\text {spa }}+d_{\mathrm{ang}}\right)}{\lambda} .
$$

Figure 4 shows the estimated combined correlation function versus $\varphi_{i-k}$ angular distances using the model described above for a pair of dipole antennas separated by $0.1,0.2$, and 0.5 spatial distances $D_{\text {spa }}$, with $D_{\text {spa }}=d_{\text {spa }} / \lambda$. Similarly, Figure 5 illustrates the estimated combined correlation function versus spatial distances $D_{\text {spa }}$ using the model described above for a pair of dipole antennas separated angularly by 30 , 90 , and 120 degrees $\left(\varphi_{i-k}\right)$.

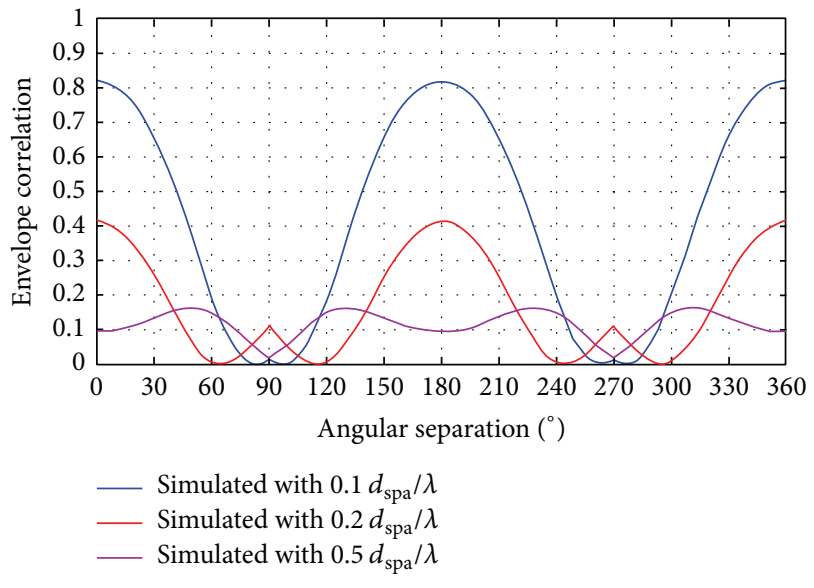

FIGURE 4: Combined correlation function versus angular difference with spatial distance as parameter.

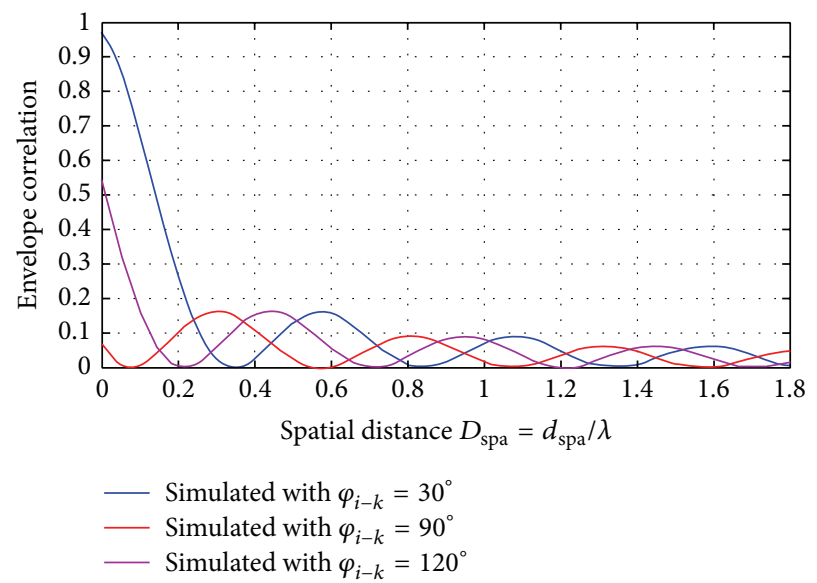

FIGURE 5: Combined correlation function versus spatial distance with angular difference as parameter. 


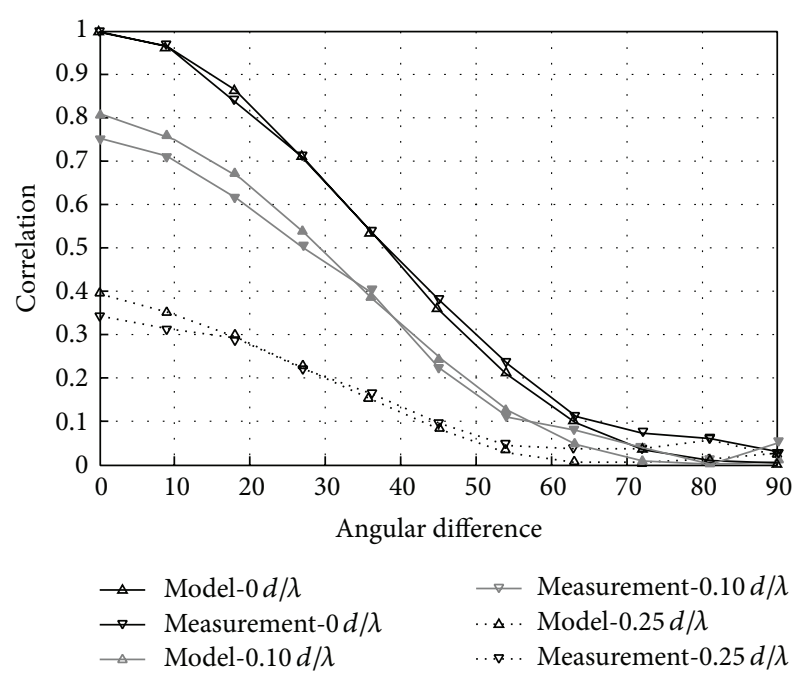

FIGURE 6: Simulated and measured correlation versus angular difference with distance as a parameter.

The model was validated through measurements that were carried out using the reverberation chamber. A comparison between measurements and the simulations using the proposed combined model is illustrated in Figure 6. It can be observed that the proposed model matches very well the performed measurements.

\section{Novel MIMO Array Schemes}

3.1. MIMO Array Configurations and Capacity. The MIMO array geometries depicted in Figure 7 with a regularly distributed number of dipoles were first studied. While it becomes difficult for a particular selection of an interelement angular separation to repeat angular distances between the first and the $n$th element, the proposed combined spatialTPD schemes really reuse states once a full rotation over $360^{\circ}$ is employed, which depends on the total number of dipole elements used. The particular different orientation of one device with respect to the next and previous one, however, is expected to result in enhanced MIMO performance.

The estimation of MIMO capacity was performed using the model proposed in [3], which requires a measurement of the MIMO correlation matrix. In [3], the correlation at the transmission side is neglected, and it is applicable when the angular spread is around 180 (all directions of arrival have the same probability). This condition does in fact resemble the isotropic behavior of the channel fading in a reverberation chamber, as the transmission antennas are typically separated by large spatial and angular distances and all directions of arrival have the same probability. The MIMO capacity thus calculated, also known as maximum allowable Shannon capacity, is defined by

$$
C_{\mathrm{MIMO}}=\log _{2}\left(\operatorname{det}\left(I_{R}+\frac{\mathrm{SNR}}{T} * R\right)\right) \text { bits } / \mathrm{s} / \mathrm{Hz} .
$$

In order to study the effect of combining TPD to spatial diversity on some novel MIMO array geometries, half-wave dipole antennas were located in fixed positions so that the correlation and capacity of the system due to the spatial diversity are constant values, and the angular interelement spacing was modified. Two different array antenna geometries, illustrated in Figure 7, were studied; dipoles were located on a circumference and on a spiral curve. In the circumference design, dipoles had the same distance to the center of the array, with a perfect symmetry. Figure 7 shows an example with 16 dipoles with a circumference radius of $0.25 d_{\text {spa }} / \lambda$. The logarithmic spiral design also depicted in Figure 7 pertains to more irregular and asymmetric noncontiguous element separations, and it is also more suitable for integration on a vertically installed base station antenna array. An equivalent spiral "radius" was defined as the distance from the farthest dipole to the centre of a two-turn spiral in order to obtain certain analogy to the circumference.

With Figure 7 and Table 1 as an example, 10 different angular reuse variations of the initial distribution of MIMO arrays consisting of 1 up to 50 dipole antennas were studied. Each new angular reuse variation was designed by assigning a specific angle value progressively among $0-360^{\circ}$ to each dipole, so that the angular distance among adjacent dipoles becomes

$$
\varphi_{\text {adj }}=\frac{360 *(n \text { th_var })}{n \_ \text {elem }}
$$

where $\varphi_{\text {adj }}$ is the angular separation among adjacent dipole antennas in the MIMO array, $n$ th_var is the angular reuse variation number, and n_elem is the number of dipole antenna elements in the MIMO array (Figure 7 and Table 1 use 16 dipoles as an example). The angular orientation of each dipole antenna in the MIMO array can then be obtained by

$$
\phi_{i}=\varphi_{\mathrm{adj}} * i
$$

where $\phi_{i}$ is the angular orientation of the $i$ th dipole in the MIMO antenna array. In this way each angular orientation is used a number of times equal to the number of angular reuses employed. As an example, Table 1 shows the angular orientations of 10 different angular reuse variations using $n_{-}$elem $=16$, that is, the same geometry systems that are shown in Figure 7. The conventional MIMO array in which only spatial diversity is employed is also listed in Table 1 and used for comparison purposes.

3.2. Evaluation of Results. MIMO capacity estimates were obtained for the different spatial geometries in Figure 7 and the 10 variations of angular reuse following the scheme of Table 1 for a number of dipole elements ranging from 1 to 50. The capacity for the conventional spatial diversity scheme without angular reuse was also estimated for comparison purposes. Results for the circumference and the spiral scenarios with a fixed radius of $0.25 d_{\text {spa }} / \lambda$ are depicted in Figures 8 and 9 , respectively, for a signal to noise ratio (SNR) of $15 \mathrm{~dB}$. The effect of using a smaller or larger radius is illustrated in Figures 10-13. Figures 10 and 11 depict the results for a circumference geometry with radius of 0.1 and $0.5 d_{\text {spa }} / \lambda$, respectively. Similarly, Figures 12 and 13 depict the results for a spiral geometry with radius of 0.1 and $0.5 d_{\text {spa }} / \lambda$, respectively. 
TABLE 1: Angular orientations of MIMO antenna array design variations $\left({ }^{\circ}\right)$.

\begin{tabular}{lcccccccccccc}
\hline \multirow{2}{*}{ Dipole element number } & Conventional & \multicolumn{3}{c}{ Combined with angular reuse in MIMO antenna array } \\
& N/A & 1 & 2 & 3 & 4 & 5 & 6 & 7 & 8 & 9 & 10 \\
\hline 1 & 0.00 & 22.50 & 45.00 & 67.00 & 90.00 & 112.50 & 135.00 & 157.50 & 180.00 & 202.50 & 225.00 \\
2 & 0.00 & 45.00 & 90.00 & 135.00 & 180.00 & 225.00 & 270.00 & 315.00 & 360.00 & 45.00 & 90.00 \\
3 & 0.00 & 67.50 & 135.00 & 202.50 & 270.00 & 337.50 & 45.00 & 112.50 & 180.00 & 247.50 & 315.00 \\
4 & 0.00 & 90.00 & 180.00 & 270.00 & 360.00 & 90.00 & 180.00 & 270.00 & 360.00 & 90.00 & 180.00 \\
5 & 0.00 & 112.50 & 225.00 & 337.50 & 90.00 & 202.50 & 315.00 & 67.50 & 180.00 & 292.50 & 45.00 \\
6 & 0.00 & 135.00 & 270.00 & 45.00 & 180.00 & 315.00 & 90.00 & 225.00 & 360.00 & 135.00 & 270.00 \\
7 & 0.00 & 157.50 & 315.00 & 112.50 & 270.00 & 67.50 & 225.00 & 22.50 & 180.00 & 337.50 & 135.00 \\
8 & 0.00 & 180.00 & 360.00 & 180.00 & 360.00 & 180.00 & 360.00 & 180.00 & 360.00 & 180.00 & 360.00 \\
9 & 0.00 & 202.50 & 45.00 & 247.50 & 90.00 & 292.50 & 135.00 & 337.50 & 180.00 & 22.50 & 225.00 \\
10 & 0.00 & 225.00 & 90.00 & 315.00 & 180.00 & 45.00 & 270.00 & 135.00 & 360.00 & 225.00 & 90.00 \\
11 & 0.00 & 247.50 & 135.00 & 22.50 & 270.00 & 157.50 & 45.00 & 292.50 & 180.00 & 67.50 & 315.00 \\
12 & 0.00 & 270.00 & 180.00 & 90.00 & 360.00 & 270.00 & 180.00 & 90.00 & 360.00 & 270.00 & 180.00 \\
13 & 0.00 & 292.50 & 225.00 & 157.50 & 90.00 & 22.50 & 315.00 & 247.50 & 180.00 & 112.50 & 45.00 \\
14 & 0.00 & 315.00 & 270.00 & 225.00 & 180.00 & 135.00 & 90.00 & 45.00 & 360.00 & 315.00 & 270.00 \\
15 & 0.00 & 337.50 & 315.00 & 292.50 & 270.00 & 247.50 & 225.00 & 202.50 & 180.00 & 157.50 & 135.00 \\
16 & 0.00 & 360.00 & 360.00 & 360.00 & 360.00 & 360.00 & 360.00 & 360.00 & 360.00 & 360.00 & 360.00 \\
\hline
\end{tabular}

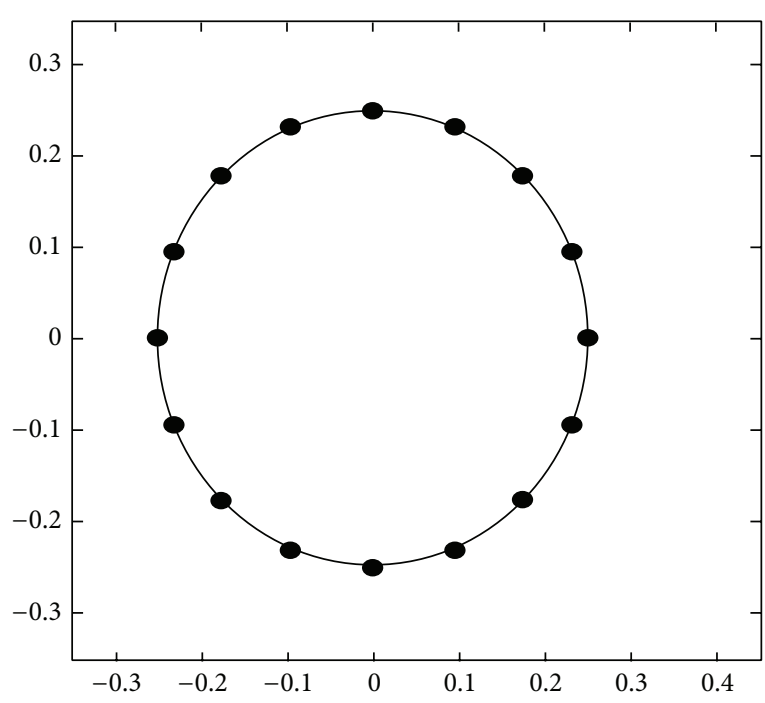

(a)

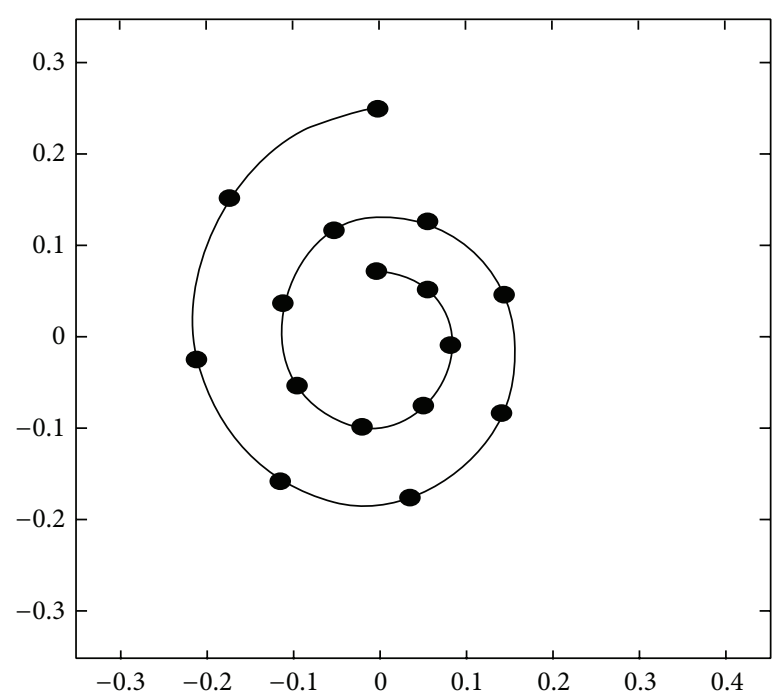

(b)

Figure 7: Circumference (a) and spiral (b) with 16 dipoles and $0.25 d_{\text {spa }} / \lambda$ radius.

\section{Analysis of Results}

A careful analysis of Figures 8-13 reveals several interesting findings. First, any combined spatial-angular scheme outperforms the conventional spatial-only diversity scheme. It is also clear from the results that the different geometries have different maximum boundaries for the capacity. In general, the circular geometry seems to be a more capacity-efficient geometry than the spiral one, yet it also takes a larger overall surface for a small number of total dipole antenna elements, while the spiral geometry can be adapted to existing vertically mounted base station antenna arrays. It is worth noting that while the antenna array with the spiral geometry may reduce the size compared with the circumference one, the MIMO capacity is also degraded, and consequently a trade-off exists between volume reduction and high capacity. In fact, the size of the array with the circumference geometry may be considered smaller than that of the spiral scenario as the antenna elements only occupy the periphery. This allows for the volume inside the circumference to be used for other purposes, like placing the electronics, for example.

It is also very clear from results that the spatial-only diversity scheme has a clear capacity limit regardless of the number of elements above a specific threshold. In other 


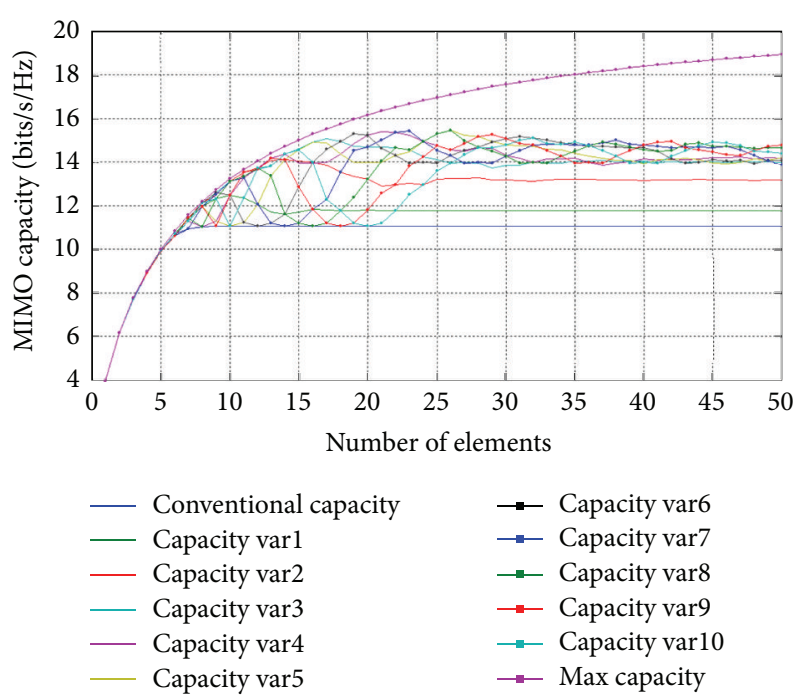

FIGURE 8: MIMO capacity for the circumference geometry with a $\mathrm{SNR}=15 \mathrm{~dB}$ and a radius of $0.25 d_{\text {spa }} / \lambda$.

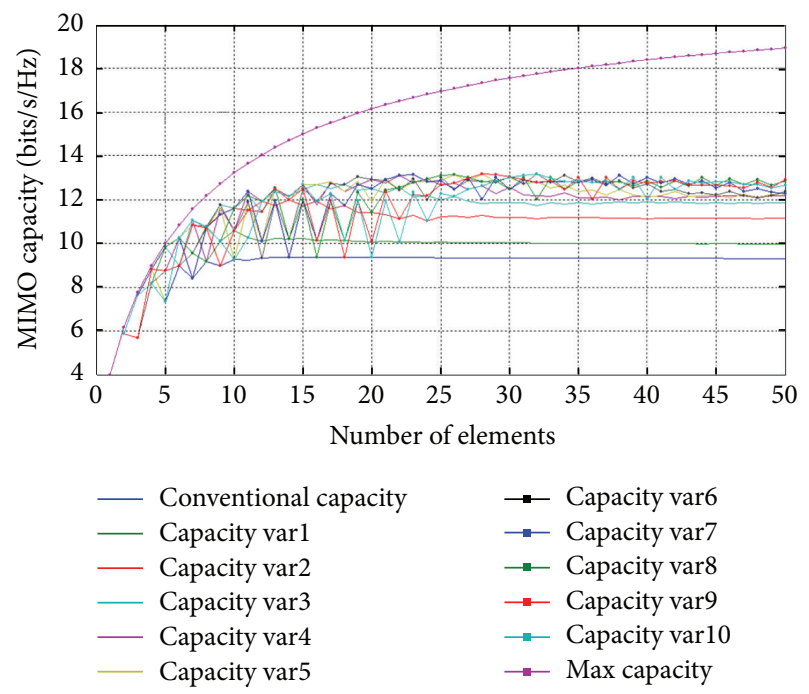

FIGURE 9: MIMO capacity for the spiral geometry with a $\mathrm{SNR}=15 \mathrm{~dB}$ and a radius of $0.25 d_{\text {spa }} / \lambda$.

words, there are a specific number of elements for the spatial-only diversity schemes above which adding additional elements does not translate into larger MIMO capacity. Likewise, it can be observed that the number of elements above which there is little improvement is smaller as the geometry radius is smaller. It is also clearly visible in all figures that true polarization diversity (TPD) serves as a perfect complement to spatial diversity in order to achieve near to maximum allowable capacities. It is equally interesting to observe that, for 30 antenna elements and beyond, the specific polarization diversity configuration that is combined with spatial diversity plays a minor role, while a higher variation in final performance can be observed when there are less than 30 antenna elements. In these situations, selecting the most

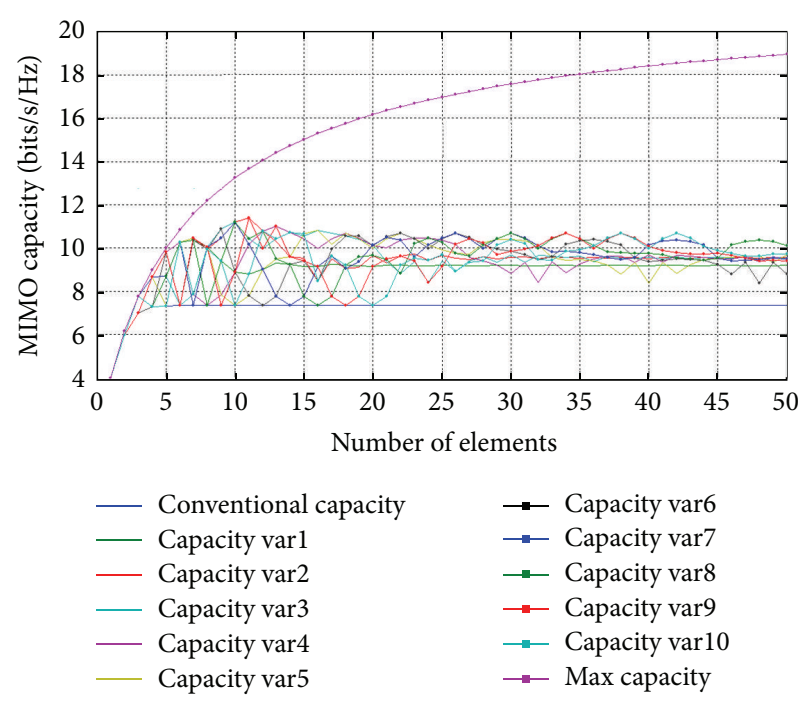

FIGURE 10: MIMO capacity for the circumference geometry with a $\mathrm{SNR}=15 \mathrm{~dB}$ and a radius of $0.1 d_{\mathrm{spa}} / \lambda$.

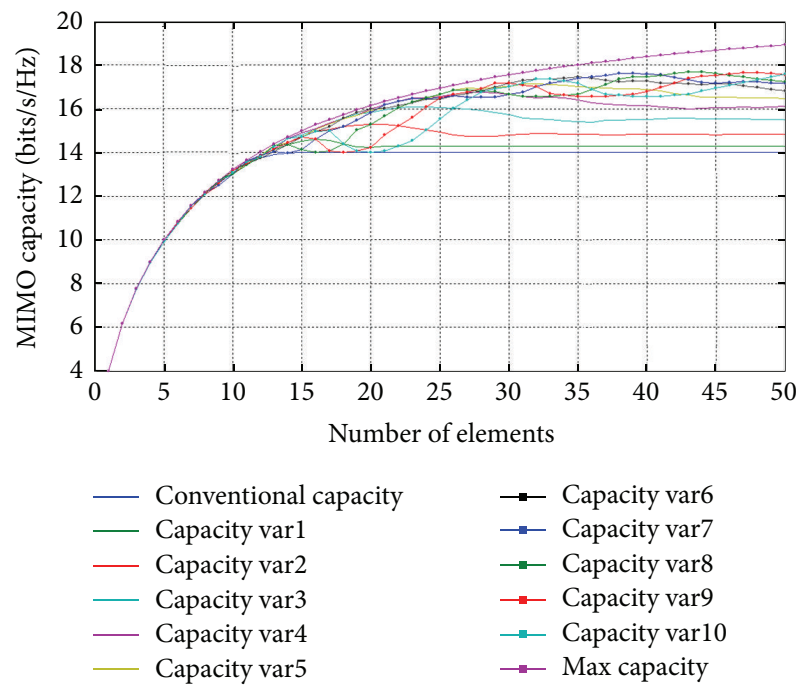

FIGURE 11: MIMO capacity for the circumference geometry with a $\mathrm{SNR}=15 \mathrm{~dB}$ and a radius of $0.5 d_{\mathrm{spa}} / \lambda$.

appropriate interelement angular separation seems to be a key factor for achieving optimum performance.

\section{Conclusions}

True polarization diversity (TPD) schemes for MIMO antenna arrays have been demonstrated to be quite useful to complement spatial diversity for achieving near to maximum allowable spectral efficiencies. While initially observed as less efficient, spiral-based geometries can achieve spectral efficiencies like more efficient circumference designs when optimizing the angular interelement spacing, which can be 


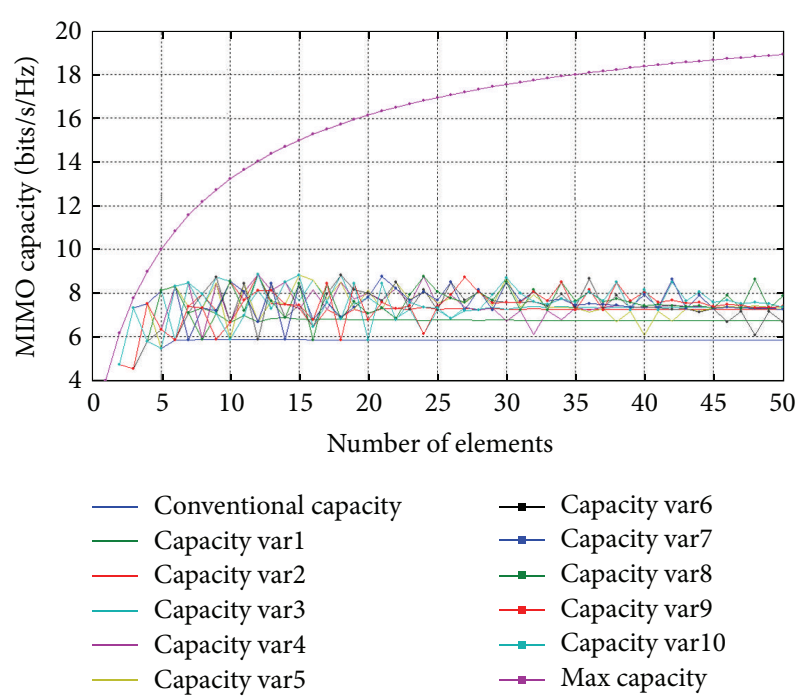

FIGURE 12: MIMO capacity for the spiral geometry with a SNR = $15 \mathrm{~dB}$ and a radius of $0.1 d_{\text {spa }} / \lambda$.

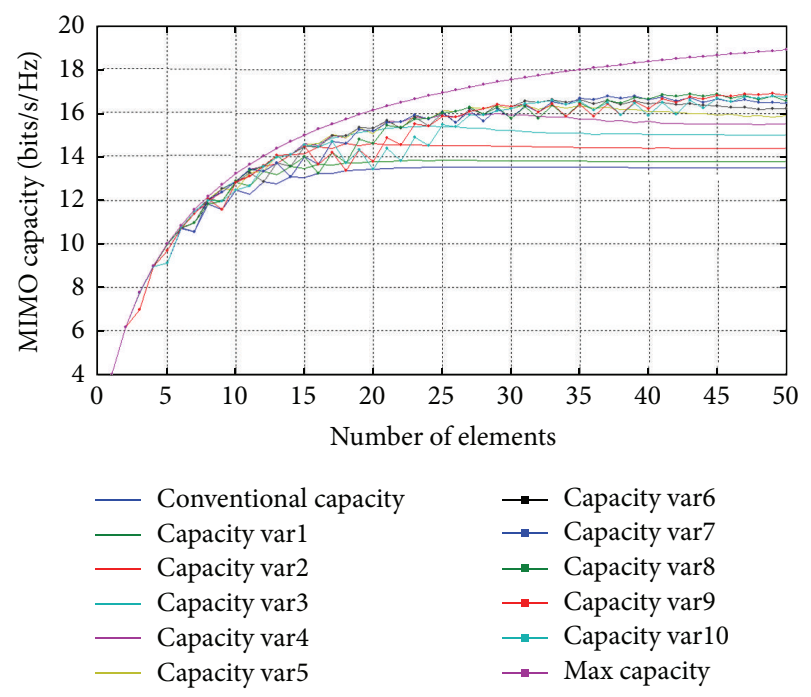

FIGURE 13: MIMO capacity for the spiral geometry with a SNR = $15 \mathrm{~dB}$ and a radius of $0.5 d_{\mathrm{spa}} / \lambda$.

useful for enhancing the MIMO performance of existing base station antenna arrays.

\section{Conflict of Interests}

The authors declare that there is no conflict of interests regarding the publication of this paper.

\section{Acknowledgments}

This work was supported in part by EMITE Ing and by Fundación Séneca, the R\&D coordinating unit of the Autonomous Region of Murcia (Spain), under Project reference 2I05SU0033.

\section{References}

[1] W. C. Jackes Jr., Microwave Mobile Communications, John Wiley \& Sons, New York, NY, USA, 1974.

[2] D. A. Hill and J. M. Ladbury, "Spatial-correlation functions of fields and energy density in a reverberation chamber," IEEE Transactions on Electromagnetic Compatibility, vol. 44, no. 1, pp. 95-101, 2002.

[3] D. A. Hill, "Linear dipole response in a reverberation chamber," IEEE Transactions on Electromagnetic Compatibility, vol. 41, no. 4, pp. 365-368, 1999.

[4] S. Loyka and G. Tsoulos, "Estimating MIMO system performance using the correlation matrix approach," IEEE Communications Letters, vol. 6, no. 1, pp. 19-21, 2002.

[5] J. F. Valenzuela-Valdés, M. Á. García-Fernández, A. M. Martínez-González, and D. A. Sánchez-Hernández, "The influence of efficiency on receive diversity and MIMO capacity for Rayleigh-fading channels," IEEE Transactions on Antennas and Propagation, vol. 56, no. 5, pp. 1444-1450, 2008.

[6] J. F. Valenzuela-Valdés, M. A. García-Fernández, A. M. Martínez-González, and D. Sánchez-Hernández, "The role of polarization diversity for MIMO systems under Rayleighfading environments," IEEE Antennas and Wireless Propagation Letters, vol. 5, no. 1, pp. 534-536, 2006.

[7] K. Rosengren and P.-S. Kildal, "Study of distributions of modes and plane waves in reverberation chambers for the characterization of antennas in a multipath environment," Microwave and Optical Technology Letters, vol. 30, no. 6, pp. 386-391, 2001.

[8] P.-S. Kildal, K. Rosengren, J. Byun, and J. Lee, "Definition of effective diversity gain and how to measure it in a reverberation chamber," Microwave and Optical Technology Letters, vol. 34, no. 1, pp. 56-59, 2002.

[9] M. A. García-Fernández, J. D. Sánchez-Heredia, A. M. Martínez-González, D. A. Sánchez-Hernández, and J. F. Valenzuela-Valdés, "Advances in mode-stirred reverberation chambers for wireless communication performance evaluation," IEEE Communications Magazine, vol. 49, no. 7, pp. 140147, 2011.

[10] P.-S. Kildal and K. Rosengren, "Correlation and capacity of MIMO systems and mutual coupling, radiation efficiency, and diversity gain of their antennas: simulations and measurements in a reverberation chamber," IEEE Communications Magazine, vol. 42, no. 12, pp. 104-112, 2004.

[11] J. F. Valenzuela-Valdés, A. M. Martínez-González, and D. A. Sánchez-Hernández, "Estimating combined correlation functions for dipoles in rayleigh-fading scenarios," IEEE Antennas and Wireless Propagation Letters, vol. 6, pp. 349-352, 2007.

[12] 3GPP TR37.977, "Verification of radiated multi-antenna reception performance of User Equipment (UE)," V 1.3.0, November 2013. 

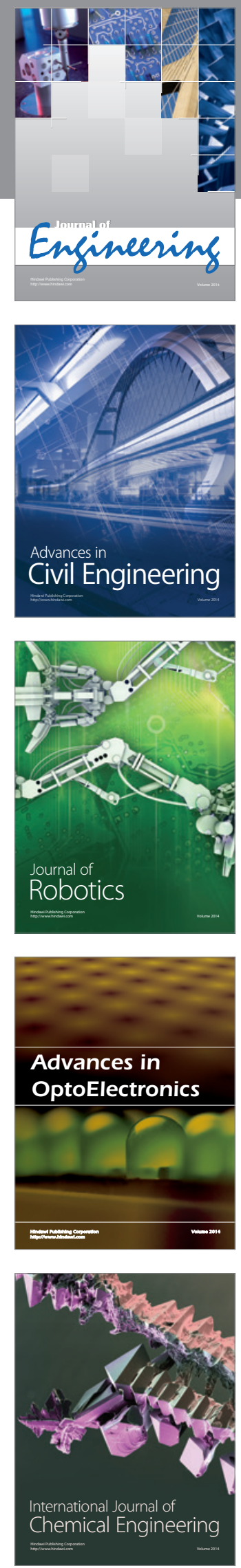

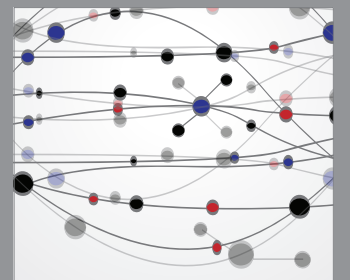

The Scientific World Journal
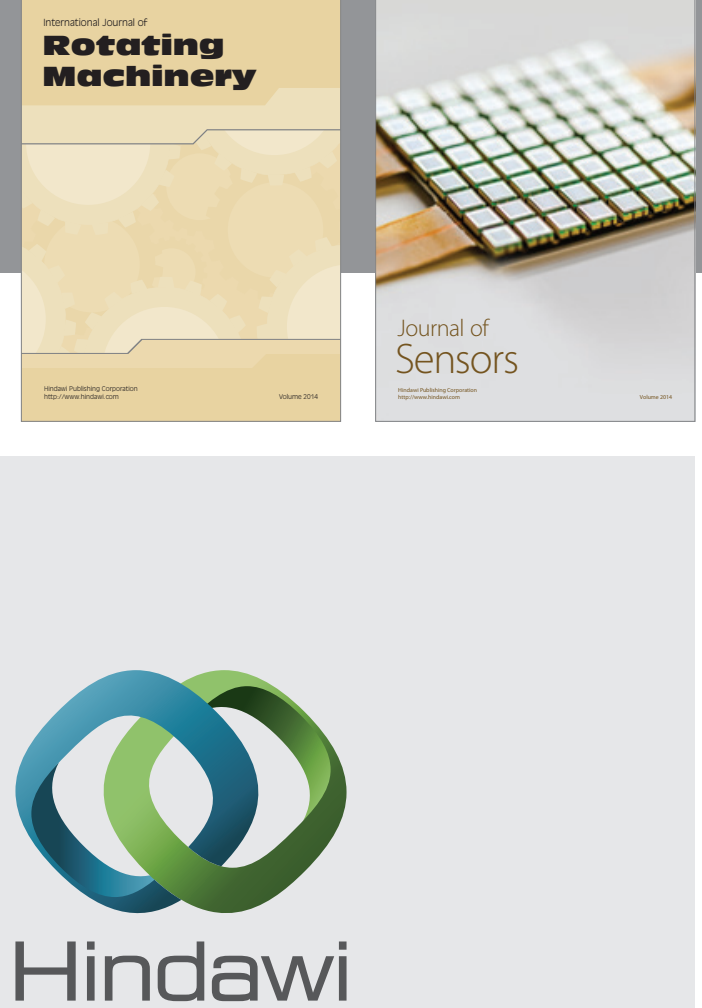

Submit your manuscripts at http://www.hindawi.com
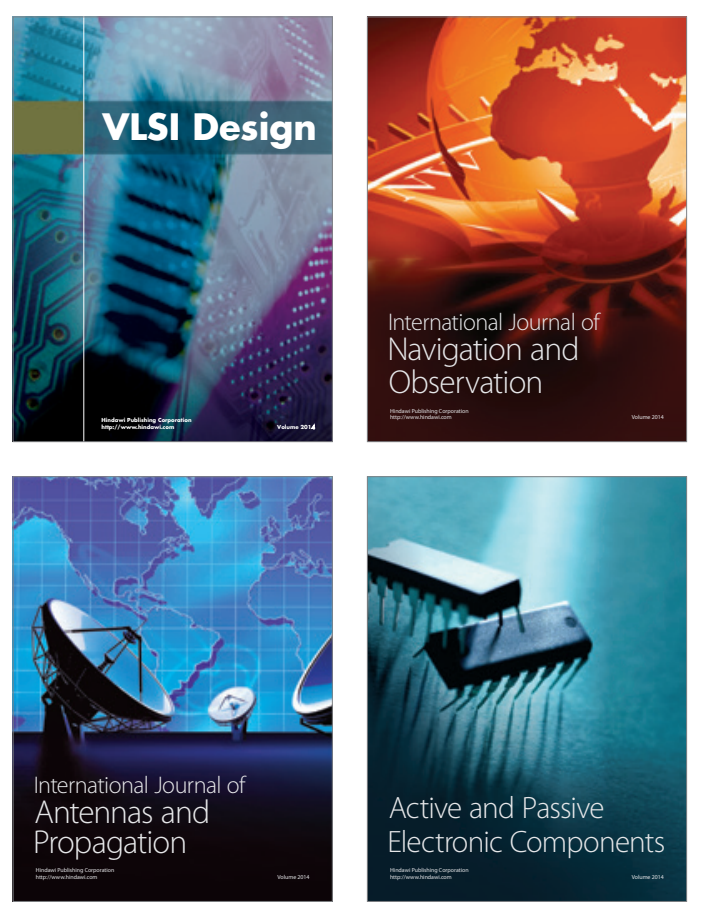
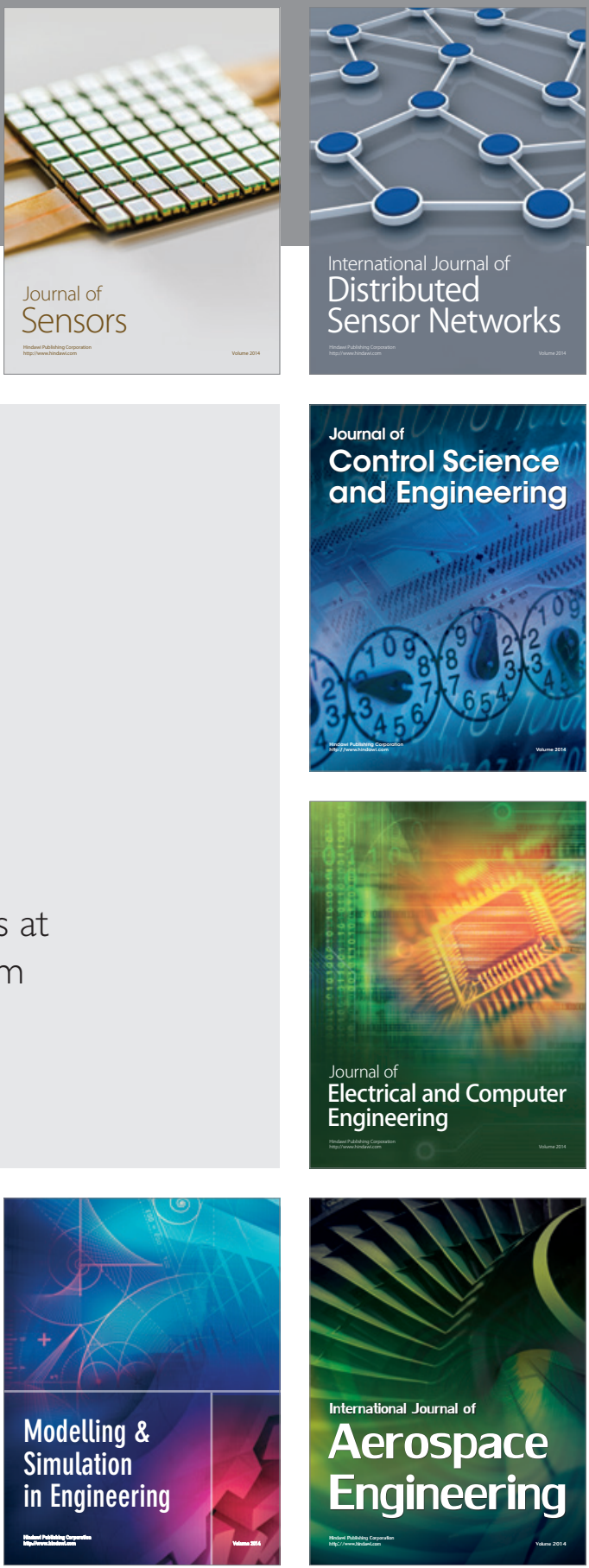

Journal of

Control Science

and Engineering
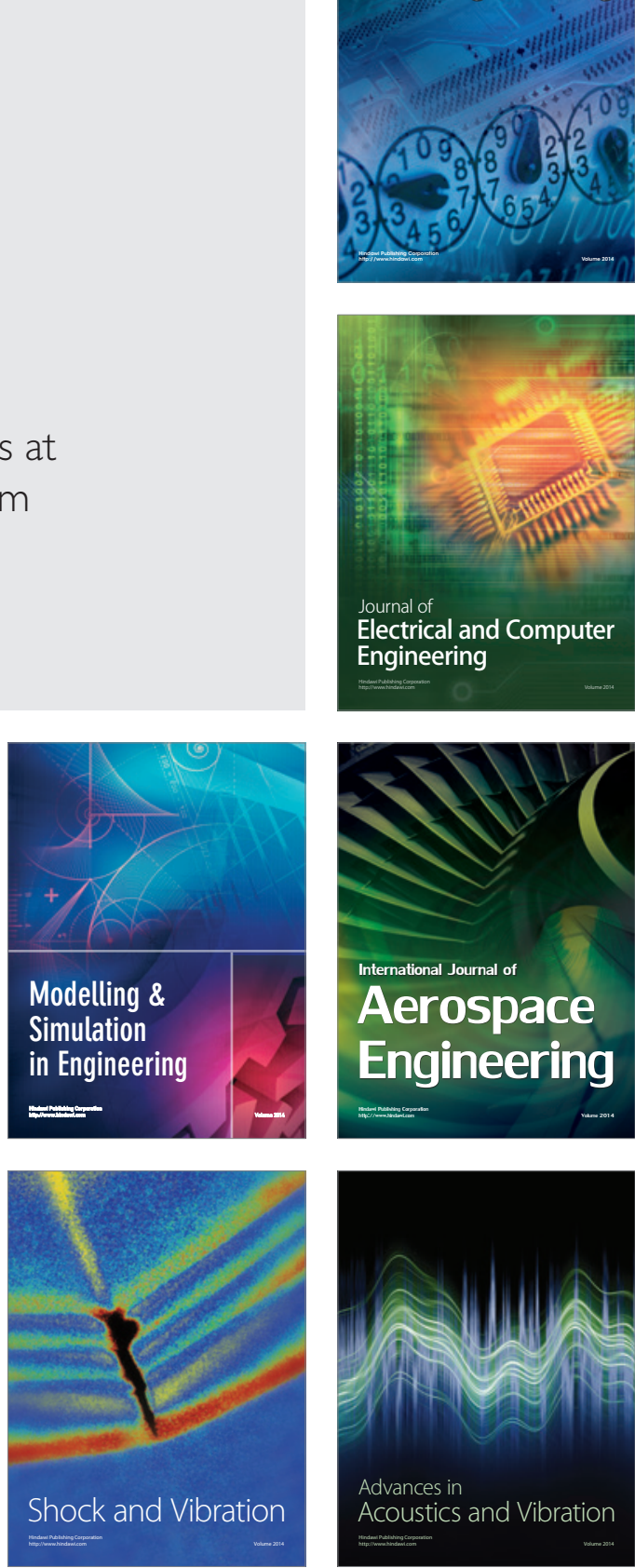